\title{
APERTURE BEAM EXPANSION BY USING A SPECTRAL 2D-GPOF METHOD
}

\author{
Massimiliano Casaletti* and Stefano Maci \\ Department of Information Engineering, University of Siena, Via \\ Roma 56, Siena 53100, Italy
}

\begin{abstract}
A method is presented for computing aperture-radiated fields in terms of complex-source type beams. These beams are generated in a natural way by expanding the aperture field spectrum in a sum of complex exponentials. The latter are obtained by using the 2D-GPOF method. Inverse transformation in spatial domain leads to an analytical form in terms of complex source points. Fields radiated by apertures obtained via this approach are validated by direct near field integration and compared with those calculated with spectralbased beam expansion which starts from the Hankel spectrum and uses a 1D-GPOF approach.
\end{abstract}

\section{INTRODUCTION}

In this paper we introduce a simple formulation for expanding aperture fields in terms of complex source point (CSP) beams [1] and discuss its potential advantages and disadvantages with respect to other formulations. In contrast with other beam expansions, here the maximum directivity of each beam is on the aperture plane. The beam expansion of an aperture-radiated field is obtained by expanding the field spectrum in the aperture plane in terms of complex exponentials. The latter are obtained by the 2D Generalized Pencil of Function 2DGPOF [2]. The original radiation integral is thus reduced to a sum of contributions, which can be identified as a CSP in spatial domain by means of the Sommerfeld identity [3]. The radiated field is thus expressed as CSP expansion where the vector complex displacements are in the aperture plane. The vector field is then obtained through the use of vector potentials.

Received 20 August 2012, Accepted 17 January 2013, Scheduled 28 January 2013

* Corresponding author: Massimiliano Casaletti (casaletti@dii.unisi.it). 
A number of different beams have been introduced in the past [1,6-16]: Gaussian beams (GB), Gaussian-ray basis functions (GRBF), higher-order Gauss-Laguerre (GLB) or Gauss-Hermite (GHB) beams, CSP, Bessel Beams (BB).

GB [6] have the limitation of satisfying Maxwell's equations only in the paraxial region; however, they desirably don't possesses branch singularity in space. GRBF [7] are obtained from GB by introducing an extra empirical parameter, with the aim to control the beam width at a given distance from the source. GL or GH beams [8-10] deal with expansions around a preferred axis of propagation with higher-order terms representing the off-axis variations. They have the advantage of constituting an orthogonal set, and therefore are often used as basis for a mode matching technique. However, their descriptive capability is still restricted to the paraxial region.

To expand the field in the whole space, CSP beams $[1,11]$ represents a good solution. Recently an exact method to express a field as a sum of complex-source points has been proposed [12]. It makes use of the Love's equivalence principle extended to a surface in the complex space enclosing the source. The continuous equivalent current distribution is then sampled obtaining a discrete number of sources radiating as a CSP. This interesting method however presents two open issues. The first one is the optimal selection of the spatial sampling rate as a function of the representation error while the second one is the choice of the optimal complex displacement for the equivalence surface.

In Gabor-type (phase-space) expansions [13-15] the field is expanded using a lattice of beams emerging from a set of points in the aperture plane and propagating from each point in a $2 \mathrm{D}$ lattice of directions. These beams describe the local radiation properties of the aperture distribution; the beam amplitudes are determined by the local radiation properties (the local spectrum) of the aperture near the lattice points. The difficulty of the implementation resides in the optimal choice of the spatial (space lattice points) and spectral (angular beam density) resolutions.

Recently, novel complex conical beams have been introduced [16]. Their aperture field expansion is obtained by applying the Generalized Pencil of Function Method (GPOF) [17-19] to the spectral domain radiation integral in cylindrical coordinates. These beams obey Maxwell's equation and, although applicable to any aperture shape, they are particularly suitable to represent circular domain aperture fields.

Different beam-expansion approaches may require a different number of beams to represent a given field. This property should 
be viewed in light of the concept of degrees of freedom introduced by Bucci and Franceschetti [20], which establishes a natural criterion for the definition of a sufficient and non-redundant number of wave functions able to fully reconstruct an arbitrary field in a given region within a predetermined error. The minimal number of wave functions needed to represent the field in the half-space above any radiating aperture is

$$
N_{\text {dof }}=2 \Sigma /(\lambda / 2)^{2}
$$

where $\Sigma$ is the area of the aperture, $\lambda$ the wavelength, and the factor 2 arises from the two independent orthogonal polarizations. Any representation requiring more beams than the number prescribed by $N_{\text {dof }}$ has some redundancy and should be improved. We emphasize that $N_{d o f}$ leads to a criterion for representing the fields without any a priori knowledge of the actual sources. If, instead, the sources are known, aperture field-matched wave objects can be found which require a number of terms much lower than $N_{d o f}$.

In the following, we will assume that the aperture field is known; however, the degrees of freedom of the aperture are taken as terms of comparison for assessing the goodness of a certain field-matched expansion.

\section{FORMULATION}

\subsection{Scalar Formulation}

Let us consider the Fourier-type spectral radiation integral in Cartesian coordinates [21]

$$
I=\frac{1}{(2 \pi)^{2}} \int_{-\infty}^{\infty} \int_{-\infty}^{\infty} \tilde{F}\left(k_{x}, k_{y}\right) \frac{e^{-j\left(k_{x} x+k_{y} y\right)-j k_{z}|z|}}{2 j k_{z}} d k_{x} d k_{y}
$$

where $\tilde{F}\left(k_{x}, k_{y}\right)$ denotes an aperture spectrum of an electric field or a scalar potential, and $e^{-j k_{z}|z|} / 2 j k_{z}$ is the spectral-domain representation of the free-space Green's function, $k_{z}=\sqrt{k^{2}-k_{x}^{2}-k_{y}^{2}}$.

The aperture spectrum $\tilde{F}$, restricted to the compact set $\left\{-k_{\max } \leq\right.$ $\left.k_{x} \leq k_{\max },-k_{\max } \leq k_{y} \leq k_{\max }\right\}$, can be expressed as a series of exponential terms by the use of the 2D-GPOF algorithm [2]:

$$
\tilde{F}\left(k_{x}, k_{y}\right)=\sum_{n=1}^{N} b_{n} e^{\left(s_{x}^{n} k_{x}+s_{y}^{n} k_{y}\right)}
$$

where the complex amplitudes $b_{n}, 2 \mathrm{D}$ complex numbers $s_{x}^{n}, s_{y}^{n}$, and the number of terms $N$ are automatically selected by the GPOF procedure. 
It has to be noted that, if the aperture has finite dimension, it is always possible to represent $\tilde{F}$ as in (3) by the use of the sampling theorem [24].

The parameter $k_{\max }$ is set a priori and truncates the evanescent portion of the aperture-field spectrum that one decides to exclude as irrelevant at the given observation level. A systematic way to choose $k_{\max }$ is presented in [24].

We observe that the aperture Fourier spectrum $\tilde{F}\left(k_{x}, k_{y}\right)$ does not necessarily be known in analytical form, but can be for instance obtained through a FFT of spatial-field samples.

Using (3) in (2) leads to

$$
I \simeq \sum_{n=1}^{N} b_{n} \frac{1}{8 \pi^{2} j} \int_{-\infty}^{\infty} \int_{-\infty}^{\infty} \frac{e^{-j\left(k_{x}\left(x+j s_{x}^{n}\right)+k_{y}\left(y+j s_{y}^{n}\right)\right)-j k_{z}|z|}}{k_{z}} d k_{x} d k_{y}
$$

which can be analytically evaluated by the use of the double spectral version of the Sommerfeld identity [3]:

$$
\frac{1}{(2 \pi)^{2}} \int_{-\infty}^{\infty} \int_{-\infty}^{\infty} \frac{e^{-j\left(k_{x} x+k_{y} y\right)-j k_{z}|z|}}{2 j k_{z}} d k_{x} d k_{y}=\frac{e^{-j k r}}{4 \pi r}=G(x, y, z)
$$

where $r=\sqrt{x^{2}+y^{2}+z^{2}}$.

The use of (5) in (4) yields

$$
I=\sum_{n=1}^{N} b_{n} G\left(x+j s_{x}^{n}, y+j s_{y}^{n}, z\right)
$$

where $G\left(x+j s_{x}^{n}, y+j s_{y}^{n}, z\right)$ can be interpreted as fields from a CPS located at the complex coordinates:

$$
\mathbf{r}_{n}^{\prime}=-j s_{x}^{n} \hat{\mathbf{x}}-j s_{y}^{n} \hat{\mathbf{y}}=\mathbf{r}_{R}^{\prime}-j a_{n} \hat{\mathbf{r}}_{C}^{\prime}
$$

where

$$
\mathbf{r}_{R}^{\prime}=\Im\left[s_{x}^{n}\right] \hat{\mathbf{x}}+\Im\left[s_{y}^{n}\right] \hat{\mathbf{y}}, a_{n}=\sqrt{\Re\left[s_{x}^{n}\right]^{2}+\Re\left[s_{y}^{n}\right]^{2}}, \hat{\mathbf{r}}_{C}^{\prime}=\frac{\Re\left[s_{x}^{n}\right] \hat{\mathbf{x}}+\Re\left[s_{y}^{n}\right] \hat{\mathbf{y}}}{a_{n}} .
$$

In order to use (7) in (5) we have to compute the complex distance

$$
r=\sqrt{\left(\mathbf{r}-\mathbf{r}_{n}^{\prime}\right) \cdot\left(\mathbf{r}-\mathbf{r}_{n}^{\prime}\right)}=R \sqrt{1+\frac{1}{R^{2}}\left(a_{n}^{2}+2 j a_{n}\left(\mathbf{r}-\mathbf{r}_{R}^{\prime}\right) \cdot \hat{\mathbf{r}}_{C}^{\prime}\right)}
$$

where $R=\left|\mathbf{r}-\mathbf{r}_{R}^{\prime}\right|$. In the far-field region (8) can be approximated as

$$
r \simeq R+j a_{n} \hat{\mathbf{R}} \cdot \hat{\mathbf{r}}_{C}^{\prime}
$$




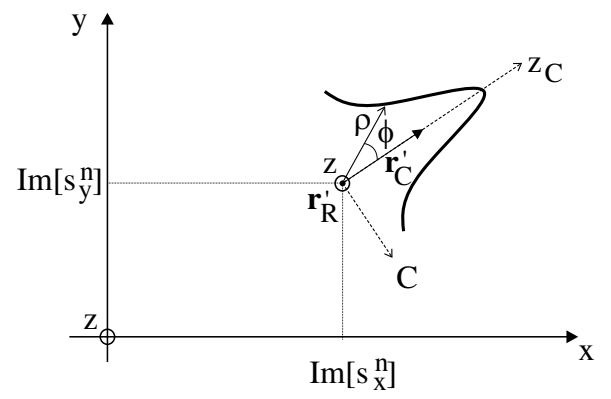

Figure 1. Field radiated by a CSP.

where $\mathbf{R}=R \hat{\mathbf{R}}=\mathbf{r}-\mathbf{r}_{R}^{\prime}$.

Using (12) in (5) leads to

$$
G\left(x+j s_{x}^{n}, y+j s_{y}^{n}, z\right)=\frac{e^{-j k R}}{4 \pi R}\left(e^{k a_{n} \hat{\mathbf{R}}^{\prime} \cdot \hat{\mathbf{r}}_{C}^{\prime}}\right)+O\left(R^{-2}\right)
$$

Equation (10) shows that the CSP beam radiates most strongly in the direction $\hat{\mathbf{r}}_{C}^{\prime}$ and most weakly $\mathrm{n}$ the direction $-\hat{\mathbf{r}}_{C}^{\prime}$. Considering a local cylindrical coordinate system $(\rho, \phi, z)$ centered in $\mathbf{r}_{R}^{\prime}$ as shown in Fig. 1, we obtain the expression

$$
G\left(x+j s_{x}^{n}, y+j s_{y}^{n}, z\right) \simeq \frac{e^{-j k R}}{4 \pi R} e^{k a_{n} \cos \phi} \simeq \frac{e^{-j k R}}{4 \pi R} e^{k a_{n}\left(1-\frac{1}{2} \phi^{2}\right)}
$$

that clearly presents the Gaussian behavior near the direction $\hat{\mathbf{r}}_{C}^{\prime}$.

In fact, in a local coordinate system with the $z$-axis aligned to $\hat{\mathbf{r}}_{C}^{\prime}$ as shown in 1, the complex distance $r$ can be approximated as

$$
r=\sqrt{\left(\rho_{c}^{2}+\left(z_{c}+j a_{n}\right)^{2}\right)} \simeq z_{c}+j a_{n}+\frac{1}{2} \frac{\rho_{c}^{2}}{\left(z_{c}+j a_{n}\right)}
$$

leading to

$$
G\left(x+j s_{x}^{n}, y+j s_{y}^{n}, z\right) \simeq \frac{1}{z_{c}+j a_{n}} e^{-j k z_{c}\left(1+\frac{1}{1} \frac{\rho_{c}^{2}}{z_{c}^{2}+a_{n}^{2}}\right)} e^{k a_{n}\left(1+\frac{1}{2} \frac{\rho_{c}^{2}}{z_{c}^{2}+a_{n}^{2}}\right)}
$$

which represents the field of a Gaussian beam with Rayleigh distance $z_{r}=a_{n}$, minimum waist size $w_{0}=\sqrt{\frac{2 a_{n}}{k}}$ and phasefront radius of curvature $R(z)=\frac{1}{z}\left(z^{2}+a_{n}^{2}\right)$.

We note that expansion (4) is accurate only within the selected spectral range $\left\{-k_{\max } \leq k_{x, y} \leq k_{\max }\right\}$. It normally happens that $\Re\left\{s_{x}^{n}\right\} \neq 0 \wedge \Re\left\{s_{y}^{n}\right\} \neq 0$, then (3) diverges exponentially for $k_{\rho}=$ $\sqrt{k_{x}^{2}+k_{y}^{2}} \rightarrow \infty\left(k_{z} \rightarrow-j k_{\rho}\right)$ in some direction of the complex plane. 
On the other hand, when (3) is inserted in (4), the integrability must be preserved over the infinite domain to yield the final representation (6). This apparent contradiction is solved under condition

$$
|z|>\max _{n}\left[\left|\Re\left\{s_{x}^{n}\right\}\right|,\left|\Re\left\{s_{y}^{n}\right\}\right|\right]
$$

namely, when the exponential attenuation term $e^{-j k_{z}|z|}$ of the spectral Green's function filters out the spectral components of (4) larger than $k_{\max }$, where $(3)$ is inaccurate. This does not present a practical limitation in most cases.

\subsection{Vector Formulation}

In order to find the vector form of (6), it is necessary to determine how (2) relates to fields or vector potentials, depending on the choice of the spectrum $\tilde{F}$. The simplest approach is to assume $\tilde{F}$ in (2) as a Cartesian spectral components of a vector potential $\mathbf{F}$ associated to twice the equivalent magnetic current at the aperture [22]; this leads to

$$
\mathbf{E}=\hat{x}\left(\frac{1}{\varepsilon} \frac{\partial F_{y}}{\partial z}\right)-\hat{y}\left(\frac{1}{\varepsilon} \frac{\partial F_{x}}{\partial z}\right)+\hat{z}\left(\frac{1}{\varepsilon}\left(\frac{\partial F_{x}}{\partial y}-\frac{\partial F_{y}}{\partial x}\right)\right)
$$

where

$$
F_{x, y}=\sum_{n} b_{n}^{x, y} W\left(x+j s_{x, n}^{x, y}, y+j s_{y, n}^{x, y}, z\right)
$$

and the coefficients $b_{n}^{x}, s_{x, n}^{x}, s_{y, n}^{x}$ and $b_{n}^{y}, s_{x, n}^{y}, s_{y, n}^{y}$ are obtained by the GPOF expansion of

$$
\begin{aligned}
& \tilde{F}_{x}=2 \tilde{E}_{y}\left(k_{x}, k_{y}\right) \\
& \tilde{F}_{y}=-2 \tilde{E}_{x}\left(k_{x}, k_{y}\right) .
\end{aligned}
$$

respectively. Finally, one has

$$
\begin{aligned}
E_{x, y} & = \pm \sum_{n} b_{n}^{y, x} \frac{\partial G\left(x+j s_{x, n}^{x, y}, y+j s_{y, n}^{x, y}, z\right)}{\varepsilon_{0} \partial z} \\
E_{z} & =\sum_{n} b_{m}^{x} \frac{\partial G\left(x+j s_{x, n}^{x, y}, y+j s_{y, n}^{x, y}, z\right)}{\varepsilon_{0} \partial y}-b_{m n}^{y} \frac{\partial G\left(x+j s_{x, n}^{x, y}, y+j s_{y, n}^{x, y}, z\right)}{\varepsilon_{0} \partial x}
\end{aligned}
$$

where the upper (lower) sign in (19) is associated to $x(y)$ component of the $E$-field.

The fields (19)-(20) can be interpreted as the field radiated by a magnetic CPS located at complex points $\mathbf{r}_{n}^{\prime}=\left(-j s_{x, n}^{x, y},-j s_{y, n}^{x, y}\right)$. 

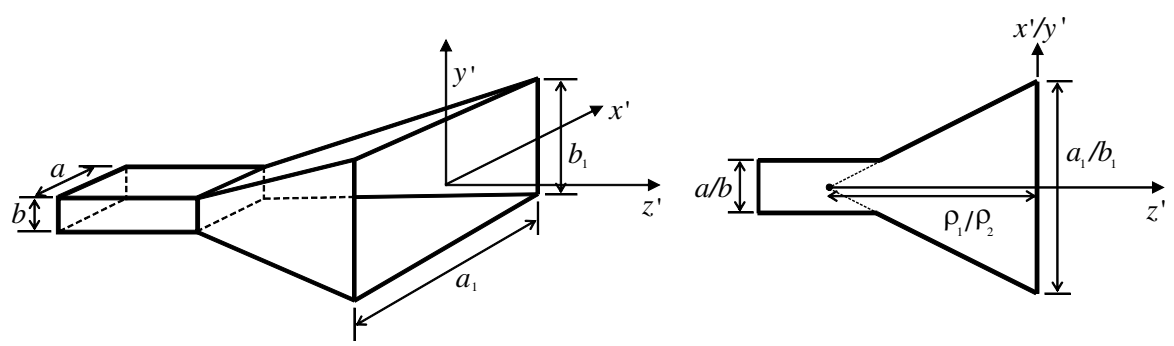

Figure 2. Geometry of the pyramidal horn antenna.

\section{EXAMPLES OF APERTURE FIELD EXPANSION}

The described approach is applied here to calculate the radiation from a pyramidal horn aperture, a rectangular phased aperture, and a circular modal aperture. The radiated field results are compared with those of a complex conical beam (CCB) expansion of type A and B formulated in [16] and a reference solution calculated by conventional aperture field integration [23].

\subsection{Pyramidal Horn}

A pyramidal horn antenna of dimensions $a_{1}=12 \lambda, b_{1}=6 \lambda, a=0.5 \lambda$, $b=0.25 \lambda, \rho_{1}=\rho_{2}=6 \lambda$ is considered (see Fig. 2). The degrees of freedom of the antenna aperture can be found using (1)

$$
N_{\text {dof }}=2 \frac{a_{1} b_{1}}{(\lambda / 2)^{2}}=576
$$

A standard analytical approximation of the Cartesian components of the aperture $y$-directed $E$-field spectrum [23] has been used for the expansion.

A total of 117 magnetic-current complex point sources have been automatically generated by the GPOF procedure. Fig. 3(a) gives a synthetic map of the complex displacement: the real part (namely $\mathbf{r}_{R}^{\prime}=\left(\Im\left\{s_{x}^{n}\right\}, \Im\left\{s_{y}^{n}\right\}\right)$ are denoted by small dots; the imaginary part of the complex position of the CPS's $\left(a_{n} \hat{\mathbf{r}}_{C}^{\prime}=\left(\Re\left\{s_{x}^{n}\right\}, \Re\left\{s_{y}^{n}\right\}\right)\right.$, related to the beam direction, by small arrows applied to the dots. Fig. 3(b) represents the phasors of the complex coefficients $b_{n}^{x}$ associated to the CPS's, and applied to the real part of the CPS position.

Figure 4 presents comparative results between the radiated electric field in the $\phi=0^{\circ} / 90^{\circ}$ cut-planes obtained by this technique and by the reference solution. Results provided by the complex conical beams 


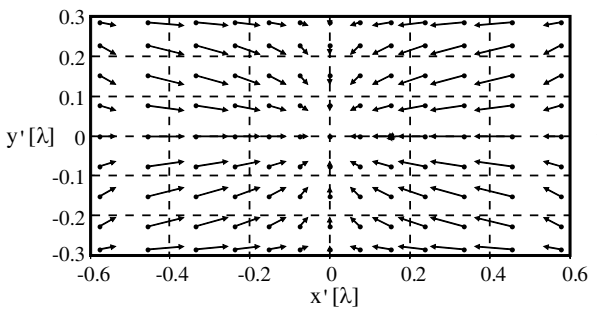

(a)

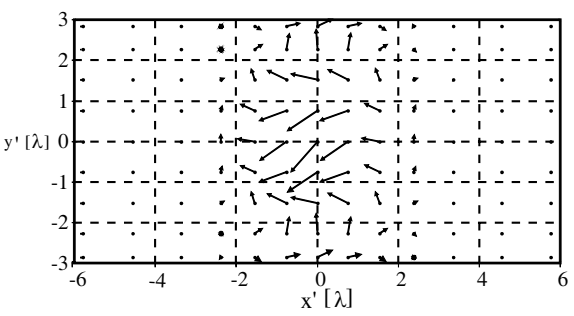

(b)

Figure 3. Aperture $12 \lambda \times 6 \lambda$ of the pyramidal horn. (a) Real parts $\left(\mathbf{r}_{R}^{\prime}\right.$, dots) and imaginary parts $\left(a_{n} \hat{\mathbf{r}}_{C}^{\prime}\right.$, arrows) of the source complex positions. (b) Phasors of the complex coefficients of the CPS aperture field expansion.

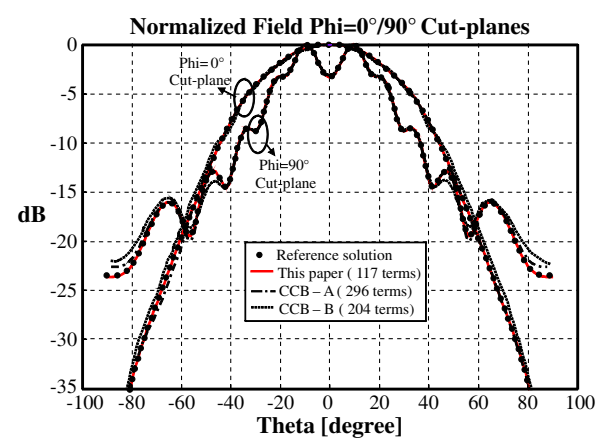

Figure 4. Pyramidal horn antenna aperture $12 \lambda \times 6 \lambda$ : $E$-plane $\left(\phi=0^{\circ}\right)$ and $H$-plane $\left(\phi=90^{\circ}\right)$ cuts of the total electric field amplitude radiated by the horn in far zone calculated with various beam expansions.

expansion (CCB) [16] type A (296 beams) and type B (204 beams) are included for comparison.

\subsection{Rectangular Aperture}

As a second example, a rectangular aperture of dimensions $5 \lambda \times 2 \lambda$ $\left(N_{\text {dof }}=80\right)$ is considered.

A first test has been made considering an aperture field which possesses a uniform amplitude and a linear phase field such as to radiate the main beam in direction $\theta_{0}=45^{\circ}, \phi_{0}=0^{\circ}$. Figures 5 (a) and $5(\mathrm{~b})$ present the map of the source complex positions and the far-field results in the $\phi=0^{\circ}$ cut-plane, respectively. Even being the 


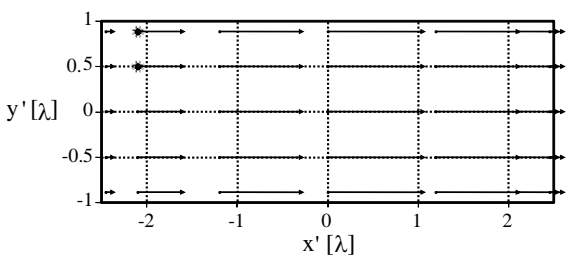

(a)

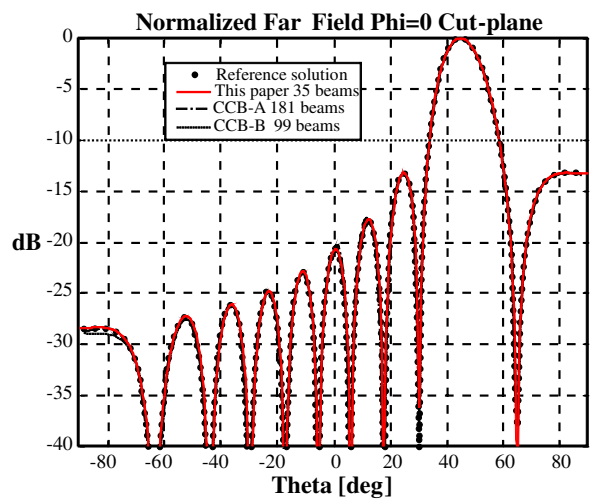

(b)

Figure 5. Rectangular phased aperture. (a) Real parts ( $\mathbf{r}_{R}^{\prime}$, dots) and imaginary parts $\left(a_{n} \hat{\mathbf{r}}_{C}^{\prime}\right.$, arrows $)$ of the source complex positions on the aperture plane. (b) $E$-plane $\left(\phi=0^{\circ}\right)$ cuts of the total electric field magnitude.

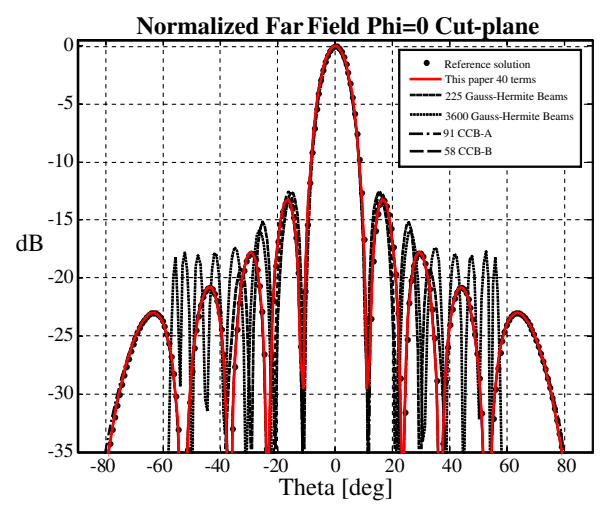

Figure 6. Rectangular uniform aperture: $E$-plane $\left(\phi=0^{\circ}\right)$ cut of the total electric field amplitude radiated in far zone calculated with various beam expansions.

accuracy, the present solution requires a lower number of beams (35 beams) with respect to CCB-A (181 beams) and CCB-B (99 beams).

A second test has been done using a uniform illumination (broadside beam) in order to compare the results also with those provided by a Gauss-Hermite (GH) expansion. The 2D-GPOF procedure give rise to 40 beams vs. 91 beams needed with CCB type A and 58 beams with CCB type B. The far-field results are shown in Fig. 6 . We observe that a GH expansion with 225 beams only matches 
the main lobe, and increasing the expansion to 3600 terms does not improve the accuracy in the overall range.

\subsection{Circular Aperture}

The Cartesian nature of the present formulation provides an excellent ability to describe aperture distribution field conformal to rectangular coordinates. However, circular aperture can be described as well at the cost of redundancy. Consider the case a circular aperture with radius $r_{w}=4 \lambda$ illuminated by a circular wave-guide $\mathrm{TM}_{44}$ mode. For this aperture size, this particular mode is the closest one to cut-off, i.e., its eigenvalue $\alpha_{44}$ is the closest to the visible region boundary, thus resulting in a fast aperture field variation.

Figure $7(\mathrm{a})$ shows the corresponding source complex positions while Fig. 7(b) presents successful comparisons among this formulation (400 beams), CCB formulation A (64 beams) and B (52 beams). The present solution needs a number of beams similar to the degrees of freedom $\left(N_{\text {dof }}=402\right)$.

As expected, the CCB are naturally more suitable for circular aperture; however, the present algorithm allows obtaining a given accuracy also for non-rectangular shapes.

Table 1 synthesizes the comparisons among the number of beams needed for the various type of representations for the various cases; $N_{d o f}$ is also reported for reference.

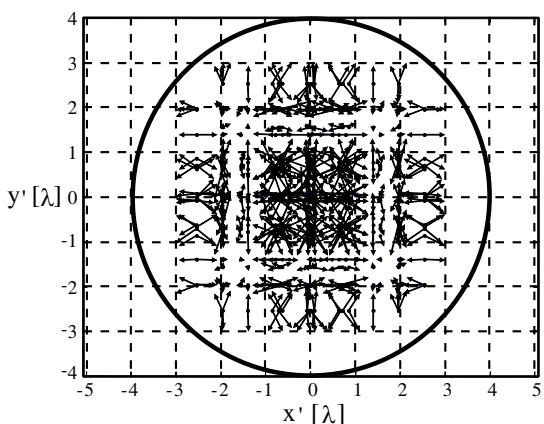

(a)

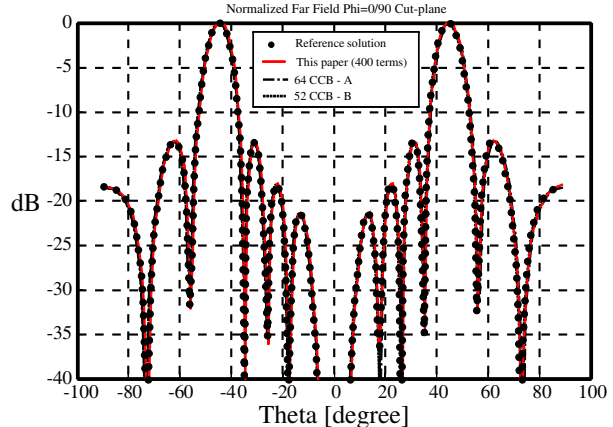

(b)

Figure 7. Circular waveguide of radius $4 \lambda$. (a) Real parts $\left(\mathbf{r}_{R}^{\prime}\right.$, dots) and imaginary parts $\left(a_{n} \hat{\mathbf{r}}_{C}^{\prime}\right.$, arrows) of the source complex positions on the aperture plane. (b) $\left(\phi=0^{\circ}\right)$ and $H$-plane $\left(\phi=90^{\circ}\right)$ cuts of the radiated total electric field magnitude by a $\mathrm{TM}_{44}$ circular waveguide mode. 
Table 1. Comparison among terms of the various expansions.

\begin{tabular}{|c|c|c|c|c|}
\hline Example & CCB-A & CCB-B & 2D-GPOF & $N_{\text {dof }}$ \\
\hline $\begin{array}{c}\text { Pyramidal Horn } \\
(12 \lambda \times 6 \lambda)\end{array}$ & 296 & 204 & 117 & 576 \\
\hline $\begin{array}{c}\text { Uniform Rectangular } \\
\text { Aperture }(5 \lambda \times 2 \lambda)\end{array}$ & 91 & 58 & 40 & 80 \\
\hline $\begin{array}{c}\text { Phased Rectangular } \\
\text { Aperture }(5 \lambda \times 2 \lambda)\end{array}$ & 181 & 99 & 35 & 80 \\
\hline $\begin{array}{c}\text { Cyrcular Aperture } \\
\mathrm{TM}_{44}(\text { Diam }=8 \lambda)\end{array}$ & 64 & 52 & 400 & 402 \\
\hline
\end{tabular}

\section{CONCLUSION}

A method for calculating aperture-radiated fields via CSP beams has been developed. The beams are obtained starting from a standard spectral domain radiation integral, upon applying 2D-GPOF expansion to the aperture field spectrum. This allows for reducing the initial double integral to a sum of CPS, whose complex displacement vector belong to the aperture plane. The numbers of beams and the complex displacement are "automatically" chosen by the 2D-GPOF spectral matching. However, whatever the aperture field is (provided it is known a priori), rectangular apertures are well-described by a number of beams lower than the $N_{d o f}$ of the aperture. Circular apertures are indeed much better described by our previous conical beam formulation [16]. We finally observe that the formulation defined here is inspired by the one discussed in [24] and based on the Shannon sampling theorem.

\section{REFERENCES}

1. Felsen, L. B., "Complex-source-point solutions of the field equations and their relation to the propagation and scattering of gaussian beams," Proc. Symp. Math., Vol. 18, 39-56, 1975.

2. Mengtao, Y., Y. Zhang, D. Arijit, J. Zhong, and T. K. Sarkar, "Two-dimensional discrete complex image method (DCIM) for closed-form Green's function of arbitrary 3D structures in general multilayered media," IEEE Trans. on Antennas and Propagat., Vol. 56, No. 5, 1350-1357, 2008. 
3. Sommerfeld, A., Partial Differential Equations, Academic Press, New York, 1964.

4. Hansen, T. B. and G. Kaiser, "Huygens' principle for complex spheres," IEEE Trans. on Antennas and Propagat., Vol. 59, No. 10, 3835-3847, 2011.

5. Tap, K., P. H. Pathak, and R. J. Burkholder, "Exact complex source point beam expansions for electromagnetic fields," IEEE Trans. on Antennas and Propagat., Vol. 59, No. 9, 3379-3390, 2011.

6. Deschamps, L. B., "The Gaussian beam as a bundle of complex rays," Electronics Letters, Vol. 7, No. 23, 684-685, 1971.

7. Chou, H. T., P. H. Pathak, and R. J. Burkholder, "Application of gaussian-ray basis functions for the rapid analysis of electromagnetic radiation from reflector antennas," IEE Proc. Microw. Antennas Propagat., Vol. 150, No. 3, 177-183, 2003.

8. Withington, S., J. A. Murphy, and K. G. Isaak, "Representation of mirrors in beam waveguides as inclined phase-transforming surfaces," Infrared Phys. Technol., Vol. 36, No. 3, 723-734, 1995.

9. Heyman, E. and I. Beracha, "Complex multipole pulsed beams and Hermite pulsed beams: A novel expansion scheme for transient radiation from well-collimated apertures," J. Opt. Soc. Am. A, Vol. 9, No. 10, 1779-1793, 1992.

10. Caravaca Aguirre, A. M. and T. Alieva, "Orbital angular moment density of beam given as a superposition of Hermite-LaguerreGauss functions," PIERS Online, Vol. 7, No. 5, 476-480, 2011.

11. Martini, E., G. Carli, and S. Maci, "A domain decomposition method based on a generalized scattering matrix formalism and a complex source expansion," Progress In Electromagnetics Research B, Vol. 19, 445-473, 2010.

12. Tap, K., P. H. Pathak, and R. J. Burkholder, "Exact complex source point beam expansion of electromagnetic fields from arbitrary closed surfaces," 2007 IEEE Antennas and Propagation Society International Symposium, 4028-4031, June 9-15, 2007.

13. Steinberg, B. Z., E. Heyman, and L. B. Felsen, "Phase space methods for radiation from large apertures," Radio Sci., Vol. 26, No. 1, 219-227, 1991.

14. Shlivinski, A., E. Heyman, A. Boag, and C. Letrou, "A phasespace beam summation formulation for ultrawide-band radiation: A multiband scheme," IEEE Trans. on Antennas and Propagat., Vol. 52, No. 8, 2042-2056, 2005.

15. Chabory, A., J. Sokoloff, and S. Bolioli, "Novel Gabor-based 
Gaussian beam expansion for curved aperture radiation in dimension two," Progress In Electromagnetics Research, Vol. 58, 171-185, 2006.

16. Skokic, S., M. Casaletti, S. Maci, and B. Srensen, "Complex conical beams for aperture field representations," IEEE Trans. on Antennas and Propagat., Vol. 59, No. 2, 611-622, 2011.

17. Hua, Y. and T. K. Arkar, "Generalized pencil-of-function method for extracting poles of an EM system from its transient response," IEEE Trans. on Antennas and Propagat., Vol. 37, No. 2, 229-234, 1989.

18. Fructos, A. L., R. R. Boix, and F. Mesa, "Efficient computation of the off-diagonal elements of the vector potential multilayered periodic dyadic Green's function," IEEE Trans. on Antennas and Propagat., Vol. 59, No. 7, 2557-2564, 2011.

19. Akyuz, M. S., V. B. Erturk, and M. Kalfa, "Closed-form Green's function representations for mutual coupling calculations between apertures on a perfect electric conductor circular cylinder covered with dielectric layers," IEEE Trans. on Antennas and Propagat., Vol. 59, No. 8, 3094-3098, 2011.

20. Bucci, O. M. and G. Franceschetti, "On the degrees of freedom of scattered fields," IEEE Trans. on Antennas and Propagat., Vol. 37, No. 7, 918-926, 1989.

21. Felsen, L. B. and N. Marcuwitz, Radiation and Scattering of Waves, Wiley-IEEE Press, Place, 1994.

22. Balanis, C. A., Advanced Electromagnetic Engineering, John Wiley and Sons, New York, 1994.

23. Balanis, C. A., Antenna Theory, 3rd Edition, John Wiley and Sons, New York, 2005.

24. Casaletti, M., S. Maci, and G. Vecchi, "A complete set of linearphase basis functions for scatterers with flat faces and for planar apertures," IEEE Trans. on Antennas and Propagat., Vol. 59, No. 2, 563-573, 2011. 